\title{
DIỀ U KHIỂN TỰ THÍCH NGHI HỆ DộNG LỰC TUYẾN TÍNH CÓ CẤU TRÚC VÀ THÔNG SỐ KHÔNG THAY DỔI
}

\author{
Vũ Chấn’ Hưng \\ Viẹn Cong nghẹ thóng tin
}

\section{Mở đầu}

Whi xây dựng các hệ thống diều khiền tự dọng trong công nghiệp, nhiều khi người ta gặp khó khăn trong việc thiết kế các bộ đî̀̂̀ chinh, do dặc tiunh dộng học của hệ thống không biết trước hoặc đặc tinh nhiêu thay đồi trong quá trình điểu khiênn. Như vậy cần phài đánh giá thông số của hệ thống và cần một bộ điểu chinh có khà năng tự định chinh thông số trong quá trỉnh điều khiên. Các hệ thống điều khiền được xây dựng theo cách này gọi là các hệ thống diều khiền thích nghi tự chinh định [1]. Bộ điều chinh tự chinh được xem như là một tổ hợp ba thành phần: bộ đánh giá thông số hệ thống. bộ xác dịnh thông số điều chinh và bộ điều chinh. Nột trong nhìng điều kiện để thông số được đánh giá hội tụ đến thông số thật là phài biết trước được bậc cuả hệ thống [1.2]. Trong nhiều tỉnh huống thực tế, bậc cuà hệ thống không biết trước. Vấn đề đánh giá thông số và điều khiền thich nghi các hệ thống có bậc không biết trước đà được một số tác già đề cập và giài quyết trong thời gian gần đây $[9,10]$. Trong bài báo này, bằng việc phát triển phương pháp biến phân $[4,5]$ lên một bước đê dánh giá thông số các đối tượng có bậc không biết trước và chọn phương pháp thiết kế bộ diểu chinh một cách thích hợp, chúng tôi đưa ra noột thuật toán đî̀̂u khiên tụ thî́ch nghi mới cho các hệ có bậc và thông số không biĉ́t trước.

\section{Phương pháp biến phân}

Phương pháp biến phân lân đầu tiên được Marchuc đề xuát đề giải bài toán 
ngược toán lí [3], được phát triçn thành phương pháp đánh giá thông số hệ thống dộng học $[4,5]$.

Cho $\Phi(k)$ là một quá trỉnh thoà măn phương trinh viết ở dạng toán tứ sau:

$$
L \Phi(k)=f(k), \Phi(0)=\Phi_{0}, k \in D \subset \mathbf{R}^{n}
$$

trong đó $L$ là một toán tự tuyến tỉnh, $k$ là biến thời gian, $f(k)$ là kích thích nguồn.

Xây dụ̂ng phương trình liên hợp với (1):

$$
L^{*} \Phi_{g}^{*}(k)=g(k)
$$

trong đó $g(k) \in L_{n}^{2}$ và $L^{*}$ là toán tư tuyến tính liên hợp với $L$, thóa mãn phương trinh sau:

$$
<L \tau, \mu>=\left\langle\tau, L^{*} \mu>\right.
$$

với mọi $\tau, \mu \in H$.

Ký hiệu $<\ldots$, > biểu thị tích vô hướng trong không gian Hilbert $H=L_{n}^{2}(D)$.

Cho một phép đo được xác định bởi một một phiếm hàm đo

$$
J_{g}[\Phi] \equiv\langle\Phi, g\rangle_{H} .
$$

Cho $D=\{0, \pm 1, \pm 2, \ldots\}$, sẽ không mất tính tông quát nếu định nghiã

$$
\left\langle\tau, \mu>=\sum_{-\infty}^{+\infty} \tau(l) \mu(l)=\sum_{l=1}^{k} \tau(l) \mu(l)\right.
$$

với $\tau(0)=0$ và $\mu(l)=0$ với mọi $l \leq 0$ và $l>k$.

Dịnh lý 1 [5]. Cho $\Phi^{\prime}$ là nghiệm của (1) với $L \equiv L^{\prime}$ v’à $f \equiv f^{\prime}$. Khi đó quan hệ giữa các biến phân $\delta J_{g}$, oL và of là nhu sau:

$$
\delta J_{g}=-<\Phi_{g}^{*}, \delta L \Phi^{\prime}>+<\Phi_{g}^{*}, \delta f>
$$

trong đó $\delta J_{g}=J_{g}^{\prime}-J_{g}, \delta L=L^{\prime}-L, \delta f=f^{\prime}-f$.

\section{Dánh giá thông số hệ động học tuyến tính có cấu trúc (bậc) không biết trước}

Phương pháp đánh giá thông số mới dựa trên công thức (5) là một phương pháp có hiệu quả $[4,5]$. Một trong các điêu kiện để thông số đánh giá hội tụ về thông số thật là phải biết rõ cấu trúc (bậc) cuả đối tượng được điều khiên. Điều này không phải bao giờ cũng được thoà mãn khi ta xây dựng các hệ điều khiên tự thích nghi trong thực tiên.

Trong bài này chúng tôi cải biến phương pháp biến phân nói trên để đánh giá thông số các hệ động học tuyến tính có bậc không biết trước. 
a. Hệ tiền định

Cho hệ tuyến tính được mô tả dưới dạng quá trỉnh ARMA

$$
\sum_{j=0}^{p_{0}} a_{J} y^{\prime}(k-j)=\sum_{j=1}^{q_{0}} b_{j} u(k-j)
$$

trong đó $a_{0}=1, a_{j}$ và $b_{j}$ là các thông số cân đánh giá, $\left(\mu_{0}, q_{0}\right)$ là bậc chưa biết trước cuả hệ thống, $u(k)$ là tín hiệu đầu vào, $y^{\prime}(k)$ là tín hiệu đầu ra cuả hệ thống.

Ký hiệu véc tơ thông số thực

$$
\theta^{*}=\left[a_{1}, a_{2}, \ldots, a_{p_{0}}, b_{1}, b_{2}, \ldots, b_{p_{0}}\right]^{T} .
$$

Đề sử dụng công thức (5) nhận dạng hệ thống (6), trước tiên cần xây dựng mô hỉnh đánh giá. Già thiết bậc cuà mô hỉnh đánh giá $\left(p_{m}, q_{m}\right)$ được chọn như sau: $p_{m} \geq$ $p_{0}, q_{m} \geq q_{0}$. Kíhi dó mô hỉnh đánh giá sẽ có clạng

$$
\sum_{j=0}^{p_{m}} \hat{a}_{j} y_{M}(k-j)=\sum_{j=1}^{q_{m}} \hat{b}_{j} u(k-j), \quad \hat{a}_{0}=1 .
$$

Điều đó có nghià là thay vỉ nhận dạng hệ (6), hệ thống có không gian thông số mở rộng sẽ là đối tượng cuà nhận dạng:

$$
\sum_{j=0}^{p_{m}} a_{j} y^{\prime}(k-j)=\sum_{j=1}^{q_{m}} b_{j} u(k-j), \quad a_{0}=1 .
$$

Ký hiệu véc tơ thông số thực mờ rộng

$$
\theta^{* \prime}=\left[a_{1}, a_{2}, \ldots, a_{p m}, b_{1}, b_{2}, \ldots, b_{q_{m}}\right]^{T} \text {. }
$$

Dề thông số được đánh giá hội tụ về thông số thự . ta đưa một kích thích ngoại vào hệ thống cũng như mô hỉnh đánh giá. Kỉch thích ngọai (ần thoà mãn điều kiện kích thích thường trực [12].

Sừ dụng định lý 1 có thể chứng minh:

Hệ quá. Cho $\Phi^{\prime}$ là nghiệm cuà $L^{\prime} \Phi^{\prime}=f^{\prime}$ với $f^{\prime} \equiv f_{0}^{\prime}+v^{\prime}$. Cho $\Phi$ là nghiệm cuà $L \Phi=f$ với $f \equiv f_{0}+v$, trong đó $v$ là kích thích ngoài, $f_{0}^{\prime}$ và $f_{0}$ là các kích thích chính. Khi đó quan hệ giữa các biến phân $\delta J_{g}, \delta L$ và $\delta f$ là như sau:

$$
\delta J_{g}=-<\Phi_{g}^{*}, \delta L \Phi^{\prime}>+<\Phi_{g}^{*}, \delta f>
$$

trong đó $\delta J_{g}=J_{g}^{\prime}-J_{g}, \delta f=f^{\prime}-f$. 
Hệ thống trong điều kiện có kích thích ngoài tác động được viết lại dưới dạng. sau:

$$
1+\sum_{j=1}^{p_{m}} a_{j} B^{j} y^{\prime}(k)=\sum_{j=1}^{q_{m}} b_{j} B^{j} u(k)+v(k), a_{0}=1
$$

trong đó $B^{j} y(k)=y(k-j), v(k)$ là kích thích ngoài.

Mô hình đánh giá tương ứng sẽ là

$$
1+\sum_{j=1}^{p_{m}} \hat{a}_{j} B^{j} y_{M}(k)=\sum_{j=1}^{q_{m}} \hat{b}_{j} B^{j} u(k)+v(k)
$$

Trên cơ sở hệ quả có thề dẫn ra phương trình nhận dạng hệ thống ở dạng cụ thề sau [8]

$$
\delta J_{g j}=\sum_{l=1}^{K} y_{g l}^{*}(l) \sum_{j=1}^{p_{m}} y^{\prime}(l-j) \delta a_{j}(k)+\sum_{l=1}^{K} y_{g l}^{*}(l) \sum_{j=1}^{p_{m}} u(l-j) \delta b_{j}(k)
$$

trong đó $y_{g j}^{*}(l)$ là nghiệm cuả phương trình liên hợp với $(10)$ :

$$
\left[1+\sum_{l=1}^{P_{m}} \hat{a}_{j}(k-1) F^{j}\right] y_{g j}^{*}(l)=g_{j}(l), F^{j} y^{*}(l)=y^{*}(l+j)
$$

và $\delta a_{j}(k)=a_{j}-\hat{a}_{j}(k-1) ; \delta b_{j}(k)=b_{j}(k-1)$.

Các phiếm hàm đo $J_{g j}^{\prime}$ và $J_{g j}$ sê là

$$
\begin{aligned}
J_{g j}^{\prime}=\left\langle g_{j}, y^{\prime}>=\sum_{l=1}^{K} g_{j}(l) y^{\prime}(l), J_{g j}^{\prime}\right. & =\left\langle g_{j}, y_{M}>=\sum_{l=1}^{K} g_{j}(l) y_{M}(l)\right. \\
\delta J_{g j} & =J_{g j}^{\prime}-J_{g j}, \quad j=1,2, \ldots, m ; m=p_{m}+q_{m}
\end{aligned}
$$

Bằng cách này ta có thề xây dựng một hệ phương trỉnh nhận dạng hệ thống.

Ký hiệu

$$
\begin{aligned}
\delta J & =\left[\delta J_{g 1}, \delta J_{g 2}, \ldots, \delta J_{g m}\right]^{T} . \\
\delta \theta & =\left[\delta a_{1}(k), \delta a_{2}(k), \ldots, \delta a_{p_{m}}(k), \delta b_{1}(k), \delta b_{2}(k), \ldots, \delta b_{q_{m}}(k)\right]^{T} .
\end{aligned}
$$

Ta có thề mô tả hệ phương trình nhận dạng ờ dạng ma trận

$$
\delta J=R \delta \theta
$$

trong đó $R$ là ma trận $m *\left(p_{m}+q_{m}\right)$ mà các phân tự cuả nó là

$$
\begin{aligned}
r_{j, i} & =\sum_{l=1}^{K} y_{g j}^{*}(l) y^{\prime}(l-i), r_{j, \dot{p}_{\dot{m}}+s}=\sum_{l=1}^{K} y_{g j}^{*}(l) u(l-s), \\
j & =1, \ldots, m ;, i=1, \ldots, p_{m} ; s=1, \ldots, q_{m} .
\end{aligned}
$$


Giài (14) bằng phương pháp bỉnh phương cı̣c tiều [1], hoặc lọc tối ưu [6] ta nhận được véc tơ biến phân thông số $\delta \theta(k)$.

Véc tơ thông số được đánh giá như sau:

$$
\dot{\theta}=\hat{\theta}(k,-1)+\delta \theta(k)
$$

b. Hệ tuyền tính ngẫu nhiên:

Cho hệ tuyến tính ngẫu nhiên:

$$
\sum_{l=0}^{p_{0}} a_{j} y^{\prime}(k-j)=\sum_{j=l}^{q_{0}} b_{j} u(k-j)+w(k)
$$

trong dó các thông 'sồ $a_{j}, b_{j}$, bậc $\left(p_{0}, q_{0}\right)$ là chưa biết, $a_{0}=1, w(k)$ là nhiễu dạng ồn trắng chulàn.

Trong 1 rường liợp này không thể dùng trực tiếp những kết quả nhận được ở phần 3-a đề dánh giá thông số hệ (16), vỉ không tính được vế trái cuả hệ (11). Vấn đề nàu đã được trỉnh bày trong $[4,5]$ và được giài quyết bằng phương pháp lọc tối ưu.

Trong phân này chúng tôi trỉnh bày một phương pháp đơn giản hơn để giải quyết bài toán bằng sư dụng bộ dự báo tối ưu một bước như một mô hỉnh đánh giá cho hệ (16).

Với cách tiếp cận đạa được trinh bày trong phần 3-a, ta xét hệ ngầu nhiên có không gian thông số mợ rộng và được kich thich bởi tín hiệu ngoài

$$
\sum_{i=0}^{p_{m}} a_{j} y^{\prime}(k-j)=\sum_{j=1}^{q_{m}} b_{j} u(k-j)+w(k)
$$

Mô hỉnh đánh giá tương ửng với (16-a) sẽ lì

$$
\left.\sum_{l=0}^{p_{m}} a_{j} y_{M I}(k-j)=\sum_{j=1}^{q_{m}} h_{j}(k-l) w k-j\right)+w(h) .
$$

Ký hiệu

$$
y_{k}=\left[y^{\prime}(1), \ldots, y^{\prime}(k)\right]
$$

Già thiết $w(k)$ và $y_{k-1}$ là các quá trỉnh độc lập.

Bổ đề. Bộ dụ báo tối uu một buớc cho mô hình đánh giá (17) có dạng sau:

$$
\grave{y}_{M}(k)=\sum_{j=0}^{p_{m}} \grave{a}_{j}(k-1) y^{\prime}(k-j)+\sum_{j=l}^{q_{m}} \hat{b}_{j}(k-1) u(k-j) .
$$


Chưng minh.

$$
\begin{aligned}
y_{M}(k) & =E\left[y_{M}(k) \mid y_{k_{-1}}\right] \\
& =E\left[\left(\sum_{j=1}^{p_{m}} a_{j}(k-1) y_{. M}(k-j)+\sum_{j=l}^{q_{m}} b_{j}(k-1) u(k-j)+w(k)\right) \mid y_{k-1}\right] \\
& =\sum_{j=1}^{p_{m}} a_{j}(k-1) E\left[y_{. M}(k-j) \mid y_{k-1}\right]+\sum_{j=l}^{q_{m}} b_{j}(k-1) E\left[u(k-j) \mid y_{k-1}\right]+E\left[(u(k)) \mid y_{k-1}\right] \\
& =\sum_{j=1}^{p_{m}} a_{j}(k-l) y^{\prime}(k-j)+\sum_{j=l}^{q_{m}} b_{j}(k-l) u(k-j)
\end{aligned}
$$

Dịnh lý 2. Cho hệ tuyén tỉnh ngẫu nhiên

$$
\sum_{j=1}^{r_{m}} a_{j}(k-l) y^{\prime}(k-j)=\sum_{j=l}^{q_{m}} \hat{b}_{j}(k-l) u(k-j)+u(k)
$$

trong dó u(k) lic nhicu dạng ôn trăng chuần. Phưong trỉnh nhận dạng cho hệ (19) có dang sau

$$
y^{\prime}(k)-y_{M}(k)=\sum_{j=1}^{r_{m}} y^{\prime}(k-j) \delta a_{j}(k)+\sum_{j=l}^{q_{m}} u(k-j) \delta b_{j}(k)+w(k)
$$

trong do yus (k) duege timh theo cong there (18).

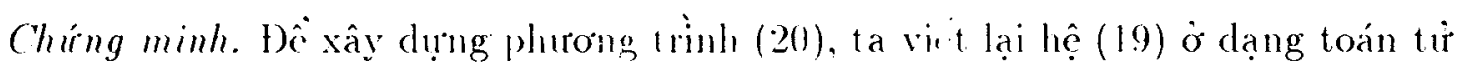

$$
I^{\prime} \Phi^{\prime}=f^{\prime}
$$

trong dó

$$
L^{\prime}=1, \quad \Phi^{\prime}=y^{\prime}(k), \quad f^{\prime}=\sum_{i=1}^{r_{m}} a_{j} y^{\prime}(k-j)+\sum_{j=\ell}^{y_{m}} b_{j} u(k-j)+u^{\prime}(k) .
$$

Ta xây dụng mô hỉnh dụ háu một bước cho hệ (20) bằng rông thị̛c (13) là bằng cách chọn

$$
L=1, \Phi=y_{M}(k) . \quad f=\sum_{j=1}^{p_{m}} a_{j}(k-l) y^{\prime}(k-j)+\sum_{j=l}^{q_{n_{1}}} b_{j}(k-l) u(k-j) .
$$

Plrương trình liên hợp turơng úng sci là

$$
y^{*}(l)=g(l)
$$


Bằng cách chọn $g(l)=1$ khi $l=k, g(l)=0$ khi $l \neq k$, vế trái cuả (20) được tính như sau

$$
\begin{aligned}
J_{g}^{\prime}=\sum_{l=1}^{k} g(l) y^{\prime}(l) & =y^{\prime}(k), \quad J_{g}=\sum_{l=1}^{k} g(l) \hat{y}_{M}(l)=\hat{y}_{M}(k) . \\
\delta J_{g} & =J_{y}^{\prime}-J_{g}=y^{\prime}(k)-y_{M}(k) .
\end{aligned}
$$

Vế phài cuà (20) sẽ là

$$
\begin{aligned}
<y^{*}, \delta f & >=\sum_{l=1}^{k} y^{*}(l)\left(\sum_{j=1}^{p_{m}} y^{\prime}(k-j) \delta a_{j}(k)+\sum_{j=l}^{q_{m}} u(k-j) \delta b_{j}(k)+w(l)\right) \\
& =\sum_{j=1}^{p_{m}} y^{\prime}(k-j) \delta a_{j}(k)+\sum_{j=l}^{q_{m}} u(k-j) \delta b_{j}(k)+w(k) .
\end{aligned}
$$

\section{Thiết kế bộ điều chinh thích nghi tự chinh định}

Bộ điều chinh tự thích nghi tự chinh định được thiết kế trên cơ sờ đặt cực hệ thống kín. Lựa chọn phương pháp thiết kế này là phù hợp, vì quá trình tính toán đơn giản, mềm dèo, đáp ứng được yêu cầu điều khiền các đối tượng được mô tà bời một quá trỉnh có bậc $\left(p_{m}, q_{m}\right)$ tùy chọn.

Viết lại hệ (16) ơ dạng sau

$$
A\left(s^{-1}\right) y^{\prime}(k)=B\left(s^{-1}\right) u(k)+w(k)
$$

trong dó

$$
\begin{aligned}
& A\left(s^{-1}\right)=1+a_{1}+\ldots+a_{p_{m}} s^{-p_{m}} \\
& B\left(s^{-1}\right)=1+b_{1}+\ldots+b_{q_{m}} s^{-q_{m}}
\end{aligned}
$$

$s^{-1}$ là toán từ dịch ngược.

Xét bọ điều chinh

$$
L\left(k-1, s^{-1}\right) u(k)=-G\left(k-1 \cdot s^{-1}\right) u^{\prime}(k)+M\left(s^{-1}\right) r(k)
$$

trong đó $\quad r(k)$ là tín hị̣̂u mầu

$$
\begin{aligned}
& L\left(k-1, s^{-1}\right)=l_{0}(k-1)+\ldots+l_{l}(k-1) s^{-l} ; l \geq q_{m}-1 \\
& G\left(k-1 . s^{-1}\right)=g_{0}(k-1)+\ldots+g_{g}(k-1) s^{-g} ; g \geq p_{m}-1
\end{aligned}
$$

$M\left(s^{-1}\right)$ là da thức tìy chọn.

Chi số thời gian $k$ chì rò các hệ số cuà đa thức có thể thay đồi theo thời gian.

Đề có được điều khiền tự thích nghi theo đặt cụce, cẩn giải phương trỉnh Diophantin

$$
A\left(k-1, s^{-1}\right) L\left(k-1, s^{-1}\right)+\hat{B}\left(k-1 . s^{-1}\right) G\left(k-1, s^{-1}\right)=T\left(s^{-1}\right)
$$


trong đó

$T\left(s^{-1}\right)$ là đa thức phản ánh các cực cuả mạch kín, được đặt theo yêu cầu.

$A\left(k-1, s^{-1}\right), \dot{B}\left(k-1, s^{-1}\right)$ là các đa thức có các hệ số là các thông số hệ thống đang được đánh giá.

Giải phương trỉnh (22) ta được các đa thức $L\left(k-1, s^{-1}\right), G\left(k-1, s^{-1}\right)$. Kết quà này được đưa vào phương trình $(21)$ để tính tín hiệu điều khiên $u(k)$. quá trình tính toán thông số cuả bộ điều chỉnh (21) trong hệ điều khiên tự thích nghi yêu cầu giải phương trỉnh Diophantin on-line bằng phương pháp truy hồi. Quá trình này được thực hiện như sau [11]:

Coi vế phải cuà hệ (22) là một quá trình động học cần đánh giá, vế trái cuả $(22)$ là mô hỉnh đánh giá tương íng. Định nghĩa hai biến ra phụ $z(k)$ và $\hat{z}(k)$

$$
\begin{aligned}
& z(k)=T(k) h(k) \\
& \dot{z}(k)=\left(\hat{A}\left(k-1, s^{-1}\right) L\left(k-1, s^{-1}\right)+\hat{B}\left(k-1, s^{-1}\right) G\left(k-1, s^{-1}\right)\right) h(k)
\end{aligned}
$$

trong đó $h(k)$ là biến vào phụ.

Ta nhận thấy, nếu $z(k)=\hat{z}(k)$ với mọi chuỗi $h(k)$ thí các hệ số cuả $L\left(k-1, s^{-1}\right), G(k-$ $\left.1, s^{-1}\right)$ khi đó là nghiệm cuà (22).

Ký hiệu

$$
\begin{aligned}
\Phi(k-1) & =\left[\hat{A}\left(k-1, s^{-1}\right) h(k-1), \ldots, \hat{A}\left(k-1, s^{-1}\right) h(k-l),\right. \\
& \left.\hat{B}\left(k-1, s^{-1}\right) h(k), \ldots, B\left(k-1, s^{-1}\right) h(k-g)\right]^{T} \\
\dot{\theta}_{r}(k-1) & =\left[\hat{f}_{l}(k-1) h(k-1), \ldots, \hat{f}_{l}(k-1) h(k-l), \hat{g}_{0}(k-1), \ldots, \hat{g}_{g}(k-1)\right]^{T}
\end{aligned}
$$

Viết lại (24) ơ dạng sau

$$
\hat{z}(k)=\Phi^{T}(k-1) \hat{\theta}_{r}(k-1)+\hat{A}\left(k-1, s^{-1}\right) h(k) .
$$

Thực hiện đánh giá quá trình (23) với mô hỉnh đánh giá (24-a) bằng phương pháp binh phương cực tiều truy hồi, ta nhận được véc tơ thông số cuả bộ điêu chinh $\hat{\theta}_{\boldsymbol{r}}(k-1)$.

Như vậy, đề xây hệ điều khiên tự thích nghi, cần hai bộ đánh giá thông số. Bộ thứ nhất gọi là bộ đáinh giá thồng số hệ thống, cung cấp các thông số cuá $\hat{A}\left(k-1, s^{-1}\right), \dot{B}\left(k-1, s^{-1}\right)$. Bộ thứ hai gọi là bộ đánh giá phụ, được sứ dụng đê tính các hệ số cuả bộ điều chinh tự thích nghi.

\section{Thuật toán điều khiền tự thich nghi}

Chọn các giá trị ban dầu $\theta(0), \hat{\theta}_{r}(0)$.

Bước 1 
xây dựng phương trỉnh nhận dạng (14) nhờ công thức (11) cho hê tiền định, hoặc nhờ công thức (20) cho hiề ngẫu nlıiên.

$B u ̛ o ̛ ́ c \stackrel{2}{2}$

Giải (14) bằng phương pháp bỉnh phương cực tiền [1], hoặc lọc tối ưu [6].

Błı́óc 3

Tinh

$$
\hat{\theta}(k)=\hat{\theta}(k-1)+\delta \theta(k) .
$$

Bước 4

Đánh giá thông số quá trình (23) với mô hỉnh (24-a) bằng phương pháp bìn $\mathrm{h}$ phương cực tiêu đề nhận được $\hat{\theta}_{r}(k)$.

Bước j

Tính tín hiệu điều khiền $u(k)$ bằng công thức (21).

Bước 6

Quay trờ lại bước 1 .

\section{Mô phóng quá trình điều khiển thích nghi tự chỉnh định}

Già sủ cần điều khiền tốc độ động cơ một chiều. Mô hỉnh toán học cuà nó có dạng sau

$$
y(k)+a_{2} y(k-2)=b_{1} u(k-1)+b_{2} u(k-2) .
$$

trong đớ véc tơ thông số $\theta^{*}=(0 ; 0.919 ; 3.45: 3.308)$ và bậc $(2,2)$ giả sư không biết trước.
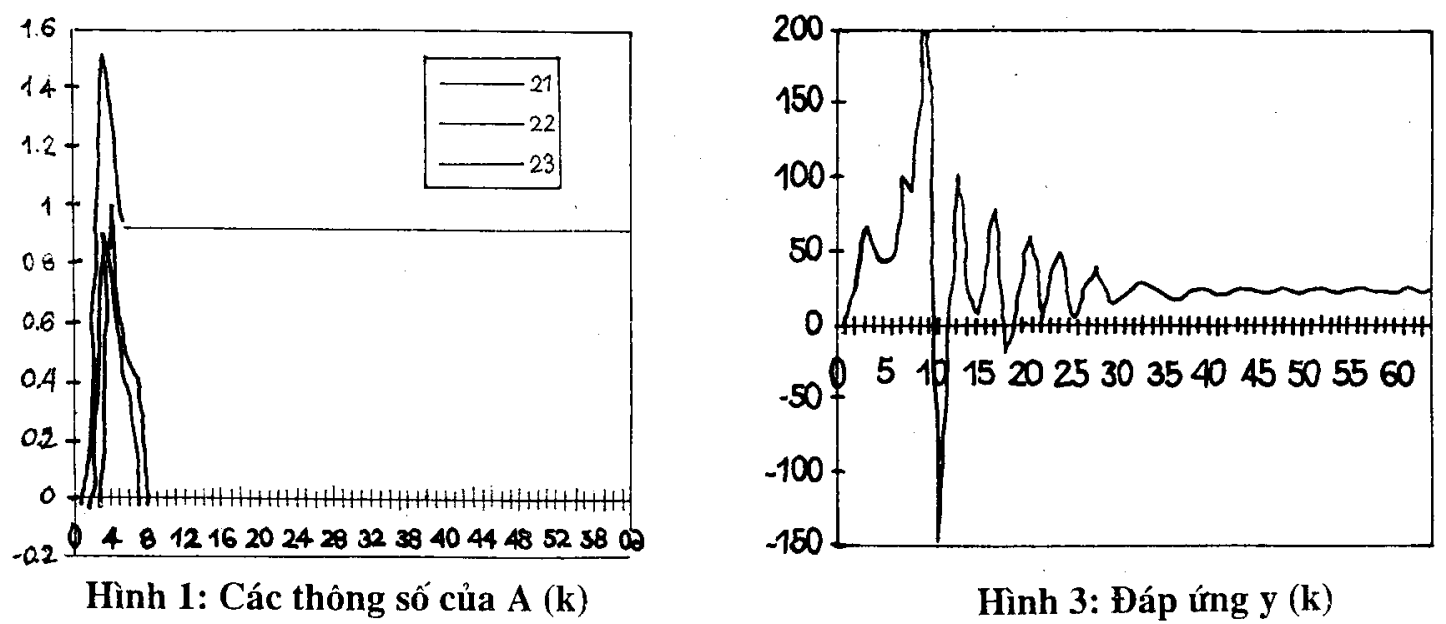

Hình 3: Đáp ứng y $(\mathrm{k})$ 
Đặc tỉnh động học cần có cuả hệ kín thề hiện qua đa thức dặc trưng $T\left(s^{-1}\right)=$ $1-0.25 s^{-2}$.

Già thiết ta chọn mô hỉnh đánh giá $(10)$ với bậc $\left(p_{m}, q_{m}\right)=(3,3)$. Bộ dịều chỉnh thích nghi dược chọn có cấu trúc tương ứng

$$
\begin{aligned}
& \hat{L}\left(k-1, s^{-1}\right)=1+\hat{l}_{1} s^{-1}+\hat{l}_{2} s^{-2}+\hat{l}_{3} s^{-3} \\
& \hat{G}\left(k-1, s^{-1}\right)=\hat{g}_{0}+\hat{g}_{1} s^{-1}+\hat{g}_{2} s^{-2}
\end{aligned}
$$

Kỉch thích ngoài được cliọn có dạng ồn trắng có cường độ $\sigma^{2}=0.1, h(k)$ là một chuỗi có biên độ bị chặn.

Hỉnh 1 chỉ ra quá trỉnh đánh giá thông số hệ thống. Các thông số được đánh giá hội tụ nhanh về các về các thông số thật cuà động cơ.

$\bar{\theta}=\left[\hat{a}_{1}, a_{2}, \hat{a}_{3}, \hat{b}_{1}, \hat{b}_{2}, \hat{b}_{3}\right]^{T}=[0.000,0.9190,0.0000,3.4500,3.3079,0.0000]^{T}$

Hình 2-a và hỉnh 2-b chì ra quá trình tự chinh định thông số cuà bộ điều chinhh. Các thông số cuà bộ điều chinh sau quá trình tự chinh có giá trị sau:

$\hat{\theta}_{r}=\left[f_{1}, f_{2}, \hat{f}_{3}, g_{0}, \dot{g}_{1}, \hat{g}_{2}\right]^{T}=[0.0720,0.0423,0.0767,0.0113,0.0777,-0.0151]^{T}$

Hỉnh 3 biều diền đầu ra cuà quá trình điều khiên tự thích nghi, với tín hiệu mẫu $r(k)=10$. Quá trình điểu khiên ôn định sau 60 bước tự chỉnh định.

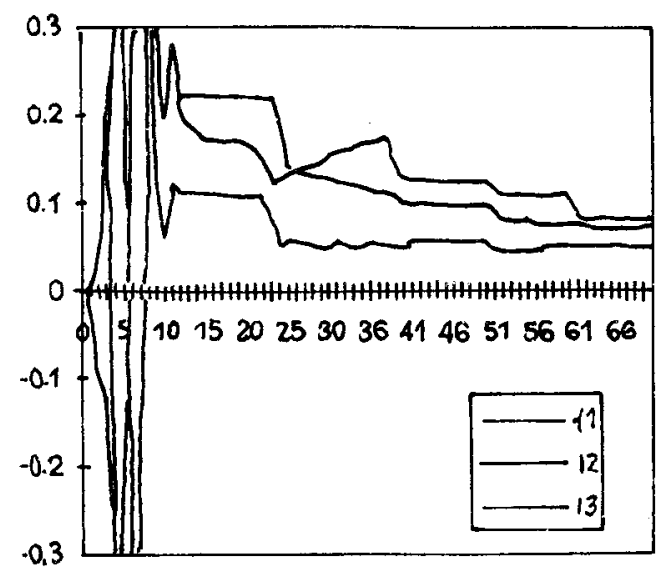

Hình 2-a: Quá trình tự chỉnh định các thông số 1

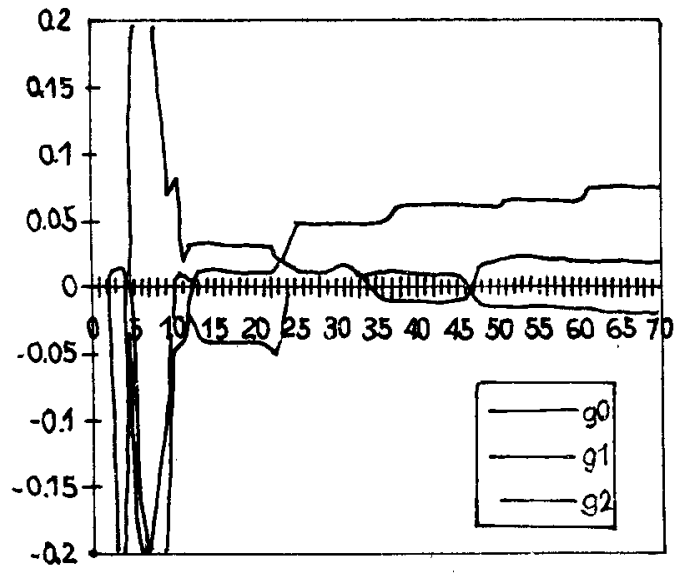

Hình 2-b: Quá trình tự chỉnh định cácthông số $\mathrm{g}$ 


\section{Tài liệu tham khaó}

1. Astrom K.J. \& Eykhoff P., A survey, Automatica v.7, 1971

2. Iserman R., Digital Control Systems, New York, 1981.

3. Marchuc G. I., Methors of computational Mathematics, Moscow, Nauka 1977.

4. Loan N. T. and Son H. H., Adaptive Paramfter Identification Method in Controlled Contanination Industries System, Proc. $5^{t h}$ Wold Filtration Congres, Vol,3 Nice, France.

5. Thoa P. H. and Son H. H., Self-tuning adaptive control based on a new parameter estimation method, Proc. IF.IC Int. Symposium on intelligent tuning and adaptive control, Singapor, 1991.

6. Loan N. T. and Son I1. H., On optimal filtering with correlated noise and singular correlation matrices, Automation and remove control, n. 5, 1982.

7. Allidin A. Y., Explicit pole-assigment self-tuning controller design, IEEF: Trans. AC, v. 34, 1988.

8. Vù Chấn Hưng \& Son H.H, Parameter and Order Estimation of Linear System based on Variational Method, Proced. of $16^{\text {th }}$ National Symposium on Theoretical Physics, 1991.

9. Chen H. F. and Zhang J. F., Identification and adaptive control for system with unknown order, delay, and coeficient, IEEF Trans. AC, v. 35, 1990.

10. Xia l. and Moor J. B., Recurcive identification of overparametrized system, IEEE Trans. Ac, v. 34, 1989.

11. LiMo and Bayoumi M. M., A novel approach to the explicite pole assigment selftuning controller design, IEEE Trans. AC, v. 34, 1989.

12. Goodwin C. C., Persistency of exitation in the presence of possibly unbounded signal, IEEE Trans. Ac v. 30, 1985.

\section{Abstract}

Self-tuning adaptive control for system with unknown order and coeeficient

A sclf-tuning adaptive control algarithm based on the modified variational method and the choosing an appropriate regulator is proposed for system with unknown order and coeeficient. 